\title{
Decreased plasma isoleucine concentrations after upper gastrointestinal haemorrhage in humans
}

\author{
C H C Dejong, W J H J Meijerink, C L H van Berlo, N E P Deutz, P B Soeters
}

\begin{abstract}
Background-A decrease in arterial isoleucine values after intragastric blood administration in pigs has been observed. This contrasted with increased values of most other amino acids, ammonia, and urea. After an isonitrogenous control meal in these pigs all amino acids including isoleucine increased, and urea increased to a lesser extent, suggesting a relation between the arterial isoleucine decrease and uraemia after gastrointestinal haemorrhage.
\end{abstract}

Methods-To extend these findings to humans, plasma amino acids were determined after gastrointestinal haemorrhage in patients with peptic ulcers $(n=9)$ or oesophageal varices induced by liver cirrhosis $(n=4)$ and compared with preoperative patients $(n=106)$.

Results-After gastrointestinal haemorrhage, isoleucine decreased in all patients by more than $60 \%$ and normalised within 48 hours. Most other amino acids increased and also normalised within 48 hours. Uraemia occurred in both groups, hyperammonaemia was seen in patients with liver cirrhosis.

Conclusions-These results confirm previous findings in animals and healthy volunteers that plasma isoleucine decreases after simulated upper gastrointestinal haemorrhage. This supports the hypothesis that the absence of isoleucine in blood protein causes decreased plasma isoleucine values after gastrointestinal haemorrhage, and may be a contributory factor to uraemia and hyperammonaemia in patients with normal and impaired liver function, respectively. Intravenous isoleucine administration after gastrointestinal haemorrhage could be beneficial and will be the subject of further research. (Gut 1996; 39: 13-17)

Keywords: human, amino acids, gastrointestinal haemorrhage, cirrhosis, peptic ulcer disease, ammonia, urea.

Surgery, Biomedica Centre/Academic

Hospital Maastricht, Maastricht, the

Netherlands

C H C Dejong

W J H J Meijerink

C L $\mathrm{H}$ van Berlo

NE P Deutz

P B Soeters

Correspondence to: Dr C H C Dejong, Department of Surgery, De Wever Hospital Heerlen PO Box 4446, NL-6401 CX Heerlen, the Netherlands.

Accepted for publication

31 January 1996 profound malaise, especially in patients with impaired liver function. ${ }^{1}$ This cannot be explained solely by the blood loss, as blood donors or even trauma patients, losing considerable amounts of blood, do not experience the characteristic malaise of patients with upper gastrointestinal bleeding. Apparently, the fact that the blood is shed into the digestive tract plays an important part in the pathophysiology and clinical picture of upper gastrointestinal haemorrhage in patients with and without liver disease:

Patients with impaired liver function often develop hepatic encephalopathy after gastrointestinal haemorrhage. ${ }^{1}$ This has been related to rising ammonia concentrations, ${ }^{1}$ probably caused by the increased nitrogen load in the presence of portasystemic shunting and liver disease, with resulting inadequate ammonia removal through urea synthesis. ${ }^{2}$ After administration of equal amounts of blood, patients with liver disease show a far greater rise in blood ammonia concentrations than patients with normal liver function. ${ }^{1}$ In this context, it has been suggested that blood is more ammoniagenic than other nitrogen sources in animals and patients with or without liver cirrhosis. ${ }^{13}$ Several explanations have been put forward for these findings, but none of them fully accounts for the 'toxic' effects of blood in the digestive tract.

In recent years, we have attempted to elucidate the ammoniagenic potential of blood in the digestive tract, by studying the effects of blood ingestion on amino acid and ammonia metabolism in pigs and healthy volunteers. ${ }^{3-5}$ Within six hours after intragastric blood administration in healthy pigs as well as after a blood pudding meal in healthy volunteers, a more than $50 \%$ decrease in the plasma values of the essential branched chain amino acid isoleucine (ILE) was observed, while the concentration of the other two branched chain amino acids leucine (LEU) and valine (VAL) and most other amino acids reached extremely high concentrations. ${ }^{3-5}$ Simultaneously, arterial urea and ammonia values increased. ${ }^{3}$ Isonitrogenous control meals led to an increase of most amino acids, including ILE, and a less pronounced increase in arterial ammonia and urea. Analysis of the amino acid composition of blood protein (mainly haemoglobin) showed an almost complete absence of the essential amino acid ILE. ${ }^{3}$ As a consequence, blood protein has a low biological value. $^{6}$

Thus, we hypothesised that the near absence of ILE in blood protein was causally related to the diminished plasma ILE concentrations as well as to the uraemia and hyperammonaemia after simulated gastrointestinal haemorrhage in experimental animals. As a logical next step, we verified this hypothesis by supplementing ILE intravenously simultaneously with intragastric blood administration in our pig model. ${ }^{4}$ The decrease in arterial ILE could be prevented and the rise in arterial urea and ammonia values could be blunted by simultaneous intravenous ILE supplementation in these pigs, suggesting improved protein and amino acid utilisation. ${ }^{4}$ 
These findings in experiments performed in otherwise healthy animals and human volunteers $^{3-5}$ could be of potential clinical relevance. If similar changes were seen after upper gastrointestinal haemorrhage in humans, this would lend support for the supplementation of ILE during upper gastrointestinal bleeding in clinical practice. This study was undertaken to further explore the applicability of this hypothesis. As a first step, we investigated whether plasma ILE also decreases after endoscopically verified upper gastrointestinal bleeding in otherwise healthy humans. In addition, four patients with liver cirrhosis were studied.

\section{Methods}

\section{Patients}

Thirteen patients with major, endoscopically verified upper gastrointestinal bleeding from peptic duodenal or gastric ulcers $(n=9$; mean (SD) age 65 (7) years (range 23-84)) or from oesophageal varices resulting from liver cirrhosis $(n=4$; mean age 43 (4) years (range 33-50)) were studied. The most important exclusion criteria were malignancies (for metabolic reasons) and, for ethical reasons, pre-existent cardiovascular disease, circulatory instability, and primary coagulation disorders. Table I lists the patient characteristics. Written informed consent was obtained from the patient or the patient's next of kin. The project was approved by the local institutional medical ethical committee.

\section{Blood sampling and determinations}

Blood for ammonia, urea, and amino acid analysis was sampled in the fasted state at the time points indicated in the Figures. The schedule was designed to sample hourly during the first six hours (starting immediately after admittance to the hospital), two hourly during the next six hours, four hourly until 24 hours, and then every 12 hours until 120 hours. If possible, a final sample was obtained 240 hours after admission. The presumed start of the bleeding, assessed by careful history taking, was taken as $\mathrm{t}=0$ hours in all cases. Antecubital venous blood was sampled from the arm opposite to the infusion site with a vacuum system (Venaject System) in $5 \mathrm{ml}$ sodiumheparin tubes (ammonia blank less than $2 \mu M)$. Samples were kept on ice during transport to the laboratory and were treated,

TABLe I Patient data

\begin{tabular}{lllll}
\hline Patient & Disease & Child's class & Sex & Age \\
\hline A & Duodenal ulcer & & male & 84 \\
B & Gastric ulcer & & male & 84 \\
C & Gastric ulcer & & female & 51 \\
D & Duodenal and gastric ulcer & & female & 79 \\
E & Gastric ulcer & & male & 73 \\
F & Gastric ulcer & & male & 23 \\
G & Duodenal ulcer & & male & 76 \\
H & Gastric ulcer & & male & 64 \\
I & Gastric ulcer & B/C & male & 50 \\
J & Alcoholic cirrhosis & C & male & 45 \\
L & Alcoholic cirrhosis & C & male & 33 \\
M & Alcoholic cirrhosis & A & male & 44 \\
& Cryptonous drug addiction & & & \\
\hline
\end{tabular}

stored at $-70^{\circ} \mathrm{C}$, and determined as detailed recently. ${ }^{48}$ Ammonia and urea were determined enzymatically and amino acids by high performance liquid chromatography technique in plasma. ${ }^{48}$ The coefficient of variance for all determinations was less than $4 \%$.

\section{Statistics}

For statistical analysis, the total sample period was divided in six intervals $(0-12 \mathrm{~h}, 12-24 \mathrm{~h}$, 24-48 h, 48-72 h, 72-96 h, 96-240 h). For each time period, the mean of all samples per patient was calculated and this mean was used for analysis.'Thus, each patient generated a maximum of six data. One way analysis of variance was used to test for main effects of time within each group. Mann-Whitney $U$ nonparametric testing was used for comparison of each time period with a control group of preoperative elective surgical patients (controls; $n=106$ ), studied simultaneously also for other purposes detailed elsewhere. ${ }^{8}$ A $\mathrm{p}<0.05$ level was considered significant.

\section{Results}

\section{General}

In most patients, the delay between the start of the bleeding and the first sample (estimated bleeding time) was approximately six hours.

Peptic ulcer patients (Figs 1 and 2, Table II) At six hours after upper gastrointestinal bleeding, ILE was only approximately $30-40 \%$ of control values in all ulcer patients (Fig 1;
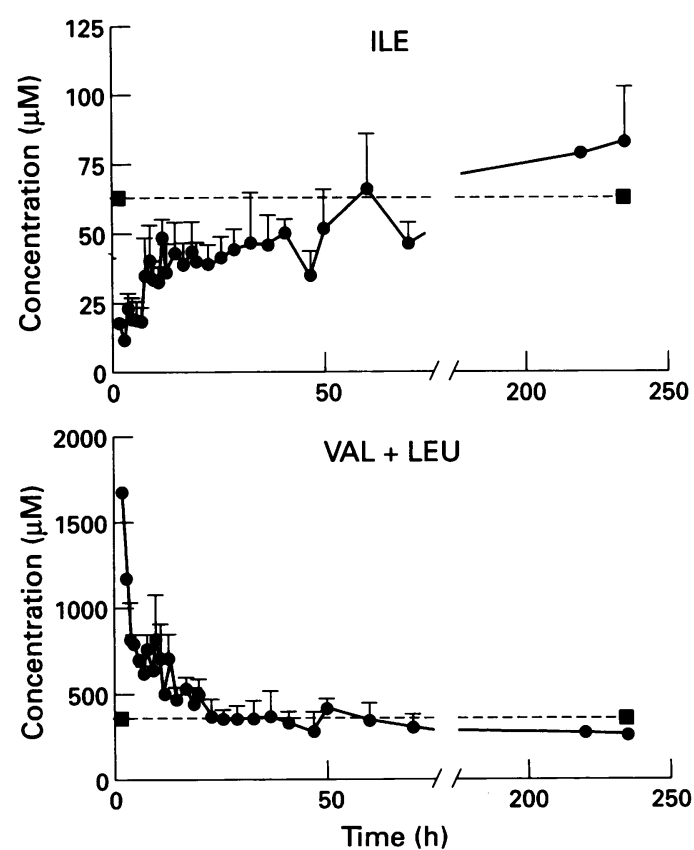

Figure 1: Plasma ILE (upper panel) and the sum of VAL and LEU (lower panel) concentrations after major, endoscopically verified, upper gastrointestinal haemorrhage in peptic ulcer patients ( $n=9$; mean age 65 years (range 23 in peptic ulcer patients $(n=9 ;$ mean age 65 years (range $23-$
$84)$ ). Data are means (SEM) $(\mu M)$. The start of bleeding, assessed by careful history taking, is taken as $t=0$ hours. The horizontal dashed line connecting filled rectangles represents normal control values of plasma ILE obtained in a group of 106 elective preoperative surgical patients. 

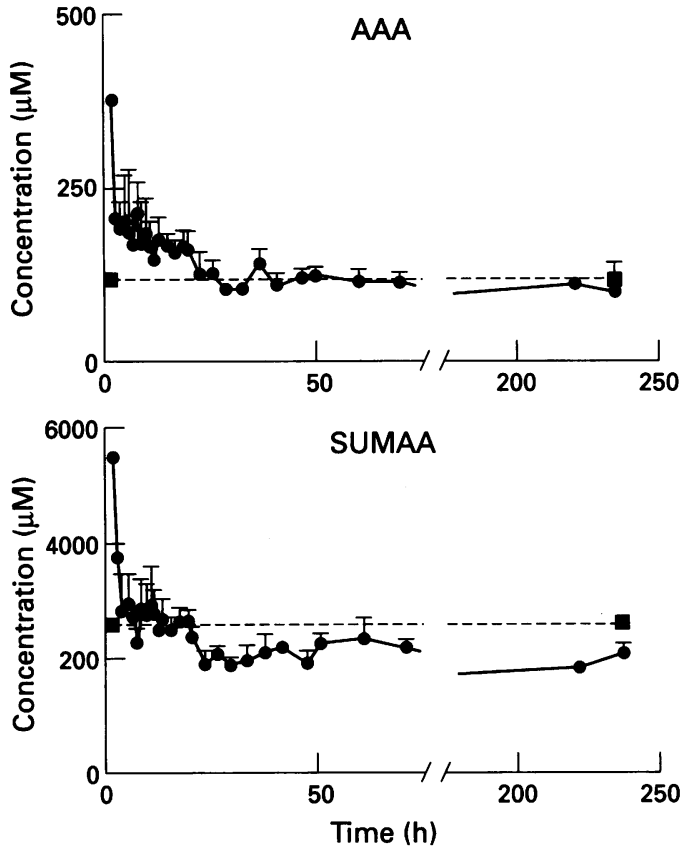

Figure 2: The aromatic amino acids ( $A A A$

(tyrosine +phenylalanine); upper panel) and the sum of the individual amino acids measured (SUMAA, lower panel) concentrations in plasma after major, endoscopically verified, upper gastrointestinal haemorrhage in peptic ulcer patients ( $n=9$; mean age 65 years (range 23-84)). Data are means (SEM) ( $\mu M)$. The start of bleeding, assessed by careful history taking, is taken as $t=0$ hours. The horizontal dashed line connecting filled rectangles represents normal control values of plasma ILE obtained in a group of 106 elective preoperative surgical patients.

$p<0.001)$. ILE increased with time $(p<0.05)$ and returned within 48 hours to values not significantly different from controls $(p>0.05)$. The other two branched chain amino acids, VAL and LEU, showed the opposite pattern: they were increased after upper gastrointestinal bleeding and normalised within 24-48 hours (Fig 1; p <0.001).

The aromatic amino acids (Fig 2) behaved identical to VAL and LEU; they were increased after upper gastrointestinal haemor- rhage compared with controls and returned to constant values within $24-48$ hours $(p<0.01)$. A similar pattern was seen for the sum of individual amino acids measured: there was a significant effect of time $(p<0 \cdot 05)$.

For the remaining amino acids, ammonia, and urea (Table II) the following patterns were seen: MET, ARG, and GLY showed a transient decrease of maximally $25 \%$ (ILE pattern). TRP values did not change in time but were decreased compared with controls. Some amino acids remained essentially unchanged (CIT, GLU, ASN, SER, THR, LYS). Other amino acids (GLN, HIS, ALA) were initially similar to control values, but decreased to stable values significantly lower than controls. The remainder ( $\alpha A B A, T A U)$ followed a pattern similar to the aromatic amino acids. Ammonia remained unchanged and urea values were strongly increased and only slowly returned to normal.

\section{Cirrhotic patients (Figs 3 and 4)}

ILE was decreased after gastrointestinal haemorrhage in cirrhotic patients and normalised within 48-72 hours. Despite the small number of patients, this decrease in ILE values was significant in the first 12 hours $(p<0.05$, nonparametric). In subject $K$ massive haemorrhage occurred in the hospital during injection of sclerosing agents for bleeding oesophageal varices and therefore no delay occurred between the start of the bleeding and the first sample. Clearly, ILE decreases to approximately $30 \%$ of initial values within 12 hours. This illustrates that the initial decrease in ILE after upper gastrointestinal bleeding is probably not seen in the other patients because of the delay between the start of the bleeding and the first sample. The sum of VAL and LEU (branched chain amino acids; Fig 4) showed a pattern similar to that seen in peptic ulcer patients; they were increased after

TABLE II Amino acid, ammonia, and urea concentrations after upper gastrointestinal haemorrhage in peptic ulcer patients

\begin{tabular}{|c|c|c|c|c|c|c|c|}
\hline Time & Control & $0-12 h$ & $12-24 h$ & $24-48 h$ & $48-72 h$ & $72-96 h$ & $96-240 h$ \\
\hline $\begin{array}{l}\text { UREA } \\
\text { AMM } \\
\text { GLU } \\
\text { ASN } \\
\text { SER } \\
\text { GLN } \\
\text { GLY } \\
\text { THR } \\
\text { HIS } \\
\text { CIT } \\
\text { ALA } \\
\text { TAU } \\
\text { ARG } \\
\text { aABA } \\
\text { TYR } \\
\text { VAL } \\
\text { MET } \\
\text { ILE } \\
\text { PHE } \\
\text { TRP } \\
\text { LEU } \\
\text { LYS } \\
\text { SUMAA } \\
\text { VAL+LEU } \\
\text { AAA } \\
\text { SUM-IIE }\end{array}$ & $\begin{array}{c}5(0) \\
25(1) \\
50(2) \\
47(1) \\
97(2) \\
664(9) \\
230(8) \\
125(3) \\
82(1) \\
35(1) \\
348(11) \\
41(1) \\
76(2) \\
19(1) \\
59(1) \\
233(4) \\
23(0) \\
63(1) \\
59(1) \\
52(1) \\
129(3) \\
157(3) \\
2586(31) \\
362(7) \\
117(2) \\
2523(30)\end{array}$ & $\begin{array}{c}19(2) \rrbracket \\
26(5) \oint \\
68(20) \\
52(6) \\
95(17) \\
584(48) \\
169(29) \oint \\
128(25) \\
87(16) \\
47(7) \\
336(65) \\
62(11) \| \\
58(6) \oint \\
35(8) \oint \\
85(17) \\
524(88) \rrbracket \\
18(2) \| \\
35(6) \rrbracket \\
107(10) \rrbracket \\
31(8) \rrbracket \\
223(49) \rrbracket \\
176(22) \\
2927(319) \\
747(135) \rrbracket \\
192(26) \rrbracket \\
2891(320)\end{array}$ & $\begin{array}{c}17(3) \| \\
24(3) \S \\
47(10) \\
52(4) \\
93(10) \\
613(53) \\
167(22) \S \\
105(12) \\
73(8) \\
38(5) \\
264(46) \S \\
53(6) \S \\
57(5) \| \\
32(6) \| \\
69(10) \\
352(44) \| \\
19(2) \S \\
43(6) \| \\
90(9) \rrbracket \\
26(3) \rrbracket \\
155(18) \\
158(16) \\
2504(166) \\
506(61) \| \\
159(16) \| \\
2461(167)\end{array}$ & 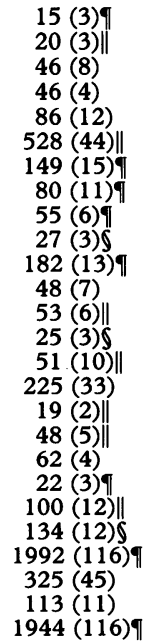 & $\begin{array}{c}11(3) \| \\
19(4) \| \\
42(8) \\
54(6) \\
109(17) \\
572(49) \\
187(18) \\
113(13) \\
55(6) 9 \\
27(4) \\
198(26) \rrbracket \\
48(9) \\
69(8) \\
23(3) \\
61(11) \\
216(32) \\
24(3) \\
58(9) \\
61(5) \\
25(5) \mathbb{1} \\
111(18) \\
162(16) \\
2216(161) \| \\
327(48) \\
122(13) \\
2158(155) \|\end{array}$ & 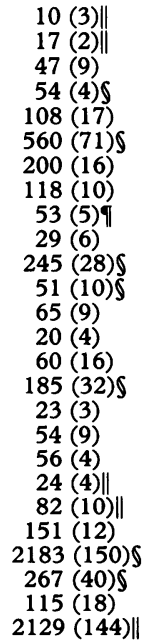 & $\begin{array}{c}9(3) \oint \\
21(3) \oint \\
53(11) \\
52(8) \\
107(26) \\
541(75) \\
202(29) \\
120(22) \\
52(8)^{\star} \| \\
32(8) \\
246(41) \\
61(14) \oint \\
69(15) \\
16(3) \\
47(1) \| \\
178(15) \neq \| \\
22(3) \\
64(8)^{\star} \\
55(2) \ddagger \\
22(4) \emptyset \\
92(8)+\| \\
132(32) \\
2163(250)^{\star} \\
270(22) \neq \| \\
102(2)+\S \\
2098(242)^{\star} \downarrow\end{array}$ \\
\hline
\end{tabular}

Data are presented as means (SEM) in $\mu \mathrm{M}$ calculated as described under Statistics, except urea (mM). Control values, obtained in preoperative elective surgical patients $(n=106)$, are given in the first column. SUMAA: sum of the individual amino acids measured; AAA: aromatic amino acids (PHE+TYR). For practical reasons, only some time points are shown in the Table. measured; AAA: aromatic amino acids (PHE + TYR). For practical reasons, only some time points are shown in the Table.
Statistics: one way analysis of variance procedure for time effects: ${ }^{\star} p<0 \cdot 05, \neq p<0 \cdot 01, \neq p<0 \cdot 001$; Mann-Whitney $U$ procedure Statistics: one way analysis of variance procedure for
versus control values $\delta \mathrm{p}<0.05, \| \mathrm{p}<0.01, \emptyset \mathrm{p}<0.001$. 

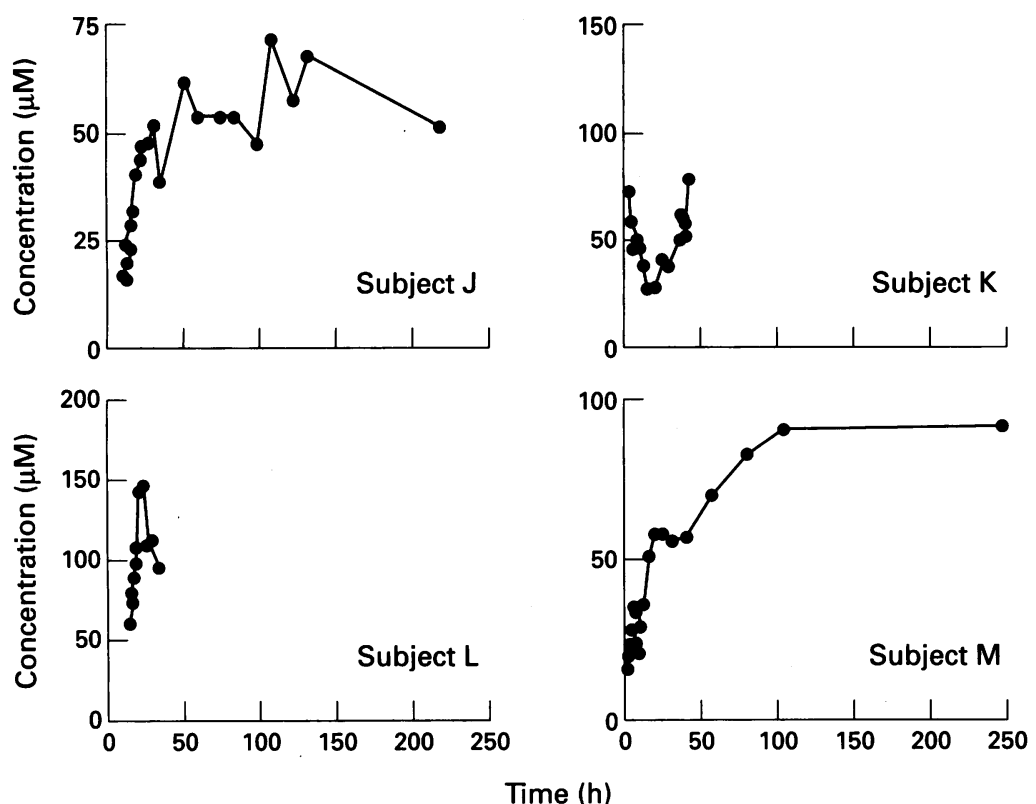

Figure 3: ILE concentrations after major, endoscopically verified, upper gastrointestinal haemorrhage in four individual cirrhotic patients (mean age 43 years (range 33-50)). The start of bleeding, assessed by careful history taking, is taken as $t=0$ hours.

gastrointestinal haemorrhage and gradually decreased to stable values. Ammonia (not shown) was increased in all cirrhotic patients after upper gastrointestinal haemorrhage and normalised after ammonia lowering treatment (lactulose and neomycin in subject $\mathrm{J}, \mathrm{K}, \mathrm{L}$, and $\mathrm{M}$, plus dopamine ${ }^{9}$ in patient $\mathrm{K}$ ).

\section{Discussion}

This study shows that ILE decreases by $60-$ $70 \%$ within six hours after gastrointestinal haemorrhage, confirming previous studies in our department on the effects of blood ingestion in pigs and healthy volunteers. ${ }^{3-5}$ This ILE decrease occurs in patients with intact liver function and in patients with impaired
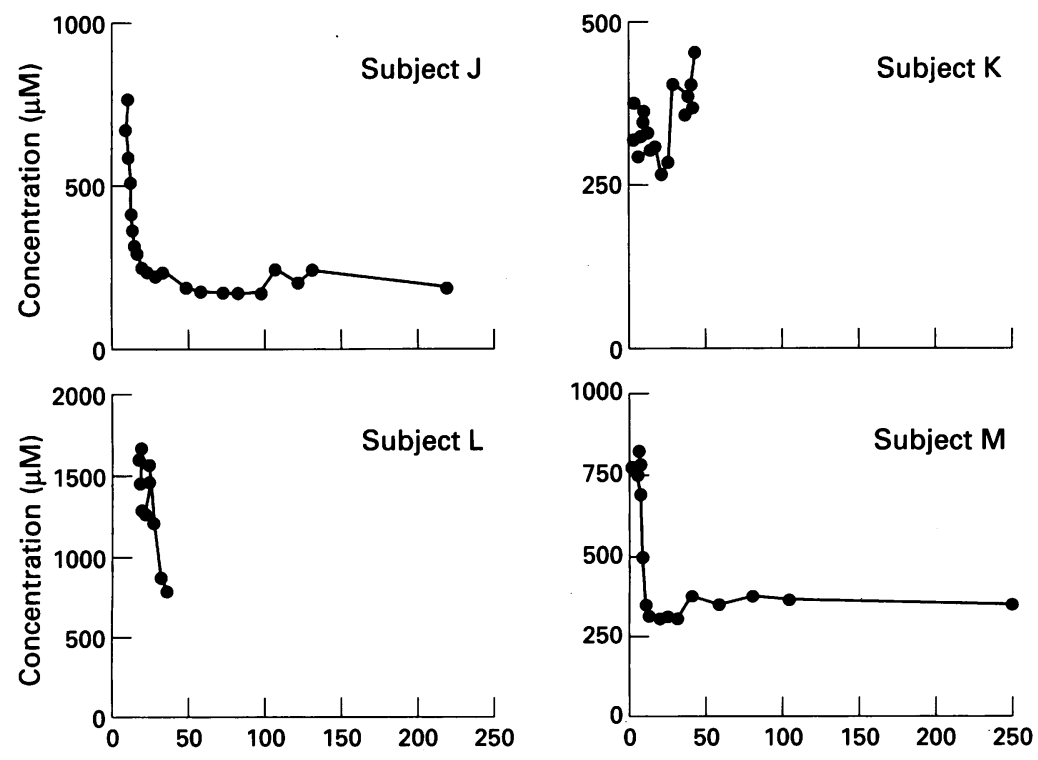

Time (h)

Figure 4: The concentration of the sum of VAL and LEU after major, endoscopically verified, upper gastrointestinal haemorrhage in four individual cirrhotic patients (mean age 43 years (range 33-50)). The start of bleeding, assessed by careful history taking, is taken as $t=0$ hours. liver function, although it is well known that branched chain amino acid metabolism is changed and plasma branched chain amino acid concentrations are decreased in the second group. ${ }^{10}$

In recent years, we have shown that within six hours after intragastric blood administration in pigs, as well as in healthy volunteers, a more than $50 \%$ decrease in plasma ILE values occurred, while the concentration of the other branched chain amino acids LEU and VAL and most other amino acids reached extremely high values. $^{3-5}$ Simultaneously, arterial urea and ammonia values increased. ${ }^{3}$ Isonitrogenous control meals led to a less pronounced increase of most amino acids, including ILE, and a significantly less pronounced increase in arterial ammonia and urea. Similar findings were also made in rats with chronic liver insufficiency. ${ }^{11}$ The changes in plasma amino acids, ammonia, and urea seen in this study are remarkably similar to those seen in previous studies in our department in experimental animals and in healthy volunteers. This seems to point to a universal pathophysiological mechanism in various species, which probably is as follows.

Blood protein (mainly haemoglobin) is almost completely devoid of ILE. ${ }^{3}$ As ILE is an essential amino acid, the absence of ILE implies that blood protein has a low biological value. ${ }^{6}$ Thus, gastrointestinal haemorrhage or blood ingestion presents the organism with a protein load that cannot be utilised for protein synthetic purposes because an essential amino acid is lacking. This may explain why most of the amino acids show a considerable and more pronounced rise after upper gastrointestinal haemorrhage than after an isonitrogenous protein load of normal composition. ${ }^{34}$ As a consequence, the constituting amino acids will be metabolised or degraded, or both, resulting in ammonia and urea synthesis. This means that the low biological value of blood protein may explain its ammoniagenic and ureagenic potential. In accordance with this, supplementing ILE simultaneously with an intragastric blood protein load should improve the biological value of blood protein and reduce its ammoniagenic and ureagenic potential.

In full agreement with this, we previously showed that the rise in arterial ammonia and urea values could be blunted in pigs by ILE infusion after blood administration intragastrically. ${ }^{4}$ In these experiments, ${ }^{4}$ the higher arterial concentrations of ammonia and several amino acids after intragastric blood administration than after a control meal $^{3}$ could be largely accounted for by increased intestinal ammonia and amino acid release into the circulation. The smaller rise in arterial ammonia and amino acids (excluding ILE) in pigs receiving ILE infusion after a blood meal could almost fully be accounted for by diminished intestinal ammonia and amino acid release. ${ }^{4}$ From these studies it was concluded that intravenous ILE supplementation improved the biological value of blood protein, seemed to improve amino acid utilisation, especially in the small bowel wall, and diminished intestinal ammonia release. ${ }^{4}$ 
The cause of the $60-70 \%$ plasma ILE decrease is speculative. It seems self evident that after a protein meal deficient in ILE, this amino acid will not increase in plasma. However, this does not explain a decrease. In blood protein, methionine is similarly deficient, ${ }^{3}$ but only decreases by $25 \%$ after gastrointestinal haemorrhage in ulcer patients, suggesting that additional factors play a part in the observed changes. Branched chain amino acid antagonism ${ }^{12-15}$ may play a part in the ILE decrease. ${ }^{4}$ Two mechanisms may be important in this respect. Firstly, the increased concentrations of VAL and LEU may compete with the decreased ILE values for transport across the cell membrane. Thus, the ILE decrease might be even more profound intracellularly. Evidence to support this has been found recently in rats with chronic liver failure, where we found a decrease in hepatic tissue ILE concentrations after intragastric blood administration. ${ }^{11}$ Secondly, increased LEU values stimulate the activity of the branched chain 2-oxoacid dehydrogenase complex, the rate limiting step in branched chain amino acid breakdown. Thus, even at these diminished ILE concentrations ILE oxidation might be increased. ${ }^{14}$

The proposed mechanism (diminished protein synthesis by ILE deficiency) is consistent with findings by others concerning impaired protein synthesis, DNA replication, cell multiplication, and cell function in an ILE deficient state. ${ }^{16-18}$ Impaired protein synthesis could shift the balance between synthesis and degradation towards degradation, inducing a net catabolic state. This can be expected to primarily affect rapidly dividing cells, short half life proteins like some clotting factors, and acute phase proteins, as well as proteins rich in ILE. In cirrhotic patients, hyperammonaemia after upper gastrointestinal bleeding may additionally impair lymphocyte function. ${ }^{19}$

The finding in pigs, that part of the adverse metabolic changes induced by blood protein administration could be reversed by intravenous ILE administration could point to possibilities for metabolic support in upper gastrointestinal bleeding patients. This could improve amino acid utilisation in protein synthetic processes and thereby lower intestinal ammonia generation. ${ }^{4}$ Diminished intestinal ammonia generation can be expected to lead to reduced urea synthesis and hence, ILE supplementation could reduce uraemia after gastrointestinal haemorrhage.

In conclusion, these data show that after upper gastrointestinal haemorrhage in otherwise healthy humans, the plasma concentration of the amino acid ILE decreases precipitously, whereas most other amino acids increase. This confirms previous studies in experimental animals and healthy volunteers. Thus, we hypothesise that, in analogy with the experimental animal situation, intravenous ILE administration during or after upper gastrointestinal bleeding could be used to improve nitrogen economy in bleeding ulcer patients. The limited data from patients with liver cirrhosis presented in this study seem to be consistent with this hypothesis. Future studies will focus on supplementing ILE during upper gastrointestinal haemorrhage in humans with and without impaired liver function.

The authors wish to thank $\mathrm{Mr} \mathrm{H}$ M H van Eijk and Mrs M A H van der Heijden for their skilled amino acid determinations.

1 Bessman AN, Mirick GS, Hawkins R. Blood ammonia levels following the ingestion of casein and whole blood. f Clin Invest 1958; 37: 990-9.

2 Rudman D, DiFulco TJ, Galambos JT, Smith RB, Salam AA, Warren WD. Maximal rates of excretion and synthesis of urea in normal and cirrhotic subjects. $\mathcal{F}$ Clin Invest 1973; 52: 2241-9.

3 van Berlo CLH, van de Boogaard AEJM, van der Heijden MAH, van Eijk HMH, Janssen MA, Bost MCF, et al. Is increased ammonia liberation after bleeding in the digestive tract the consequence of complete absence of isoleucine in hemoglobin? A study in pigs. Hepatology 1989; 10: 315-23.

4 Deutz NEP, Reijen PLM, Bost MCF, van Berlo CLH, Soeters PB. Modification of the effects of blood on amino acid metabolism by intravenous isoleucine. Gastroenterology 1991; 101: 1613-20.

5 van Berlo CLH, Dejong CHC, Meijerink WJHJ, Fick TE, von Meyenfeldt MF, Deutz NEP, et al. A decrease in plasma isoleucine levels after simulated upper digestive tract bleeding in man [Abstract]. Clin Nutr 1991; 10 supple 2: 056 .

6 Newsholme EA, Leech AR. Biochemistry for the medical sciences. New York: John Wiley, 1983.

7 van Eijk $\mathrm{HMH}$, van der Heijden MAH, van Berlo CLH, Soeters PB. Fully automated liquid-chromatographic determination of amino acids. Clin Chem 1988; 34: 2510-3.

8 van Eijk HMH, Dejong CHC, Deutz NEP, Soeters PB. Influence of storage conditions on normal amino acid concentrations. Clin Nutr 1994; 13: 374-80.

9 Zieve L, Doizaki WM, Derr RF. Reversal of ammonia coma in rats by L-DOPA: a peripheral effect. Gut 1979; 20: 28-32.

10 Weber FL, Bagby BS, Licate L, Kelsen SG. Effects of branched chain amino acids on nitrogen metabolism in patients with cirrhosis. Hepatology 1990; 11: 942-50.

11 Olde Damink SWM, Dejong CHC, Deutz NEP, Soeters PB. Intestinal and hepatic tissue isoleucine levels after simulated upper gastrointestinal hemorrhage in two models of chronic liver insufficiency in rats. [Abstract] Clin Nutr 1993; 12 (suppl 2): 43.

12 Rogers QR, Spolter PD, Harper AE. Effect of leucineisoleucine antagonism on plasma amino acid pattern of rats. Arch Biochem Biophys 1962; 97: 497-504.

13 Oestemer GA, Hanson LE, Meade RJ. Leucine-isoleucine interrelationship in the young pig. F Anim Sci 1973; 36: 674-8.

14 Aftring RP, Block KP, Buse MG. Leucine and isoleucine activate skeletal muscle branched-chain $\alpha$-keto acid dehydrogenase in vivo. Am $\Im$ Physiol 1986; 250: E599604 .

15 Swendseid ME, Villalobos J, Figueroa WS, Drenick EJ. The effects of test doses of leucine, isoleucine or valine on plasma amino acid levels. The unique effect of leucine. Am f Clin Nutr 1965; 17: 317-21.

16 Bergström J, Fürst P, Vinnars E. Effect of a test meal, without and with protein, on muscle and plasma free amino acids. Clin Sci 1990; 79: 331-7.

17 Kohn A. Differential effects of isoleucine deprivation on cell motility, membrane transport and DNA synthesis in NIL 8 hamster cells. Exp Cell Res 1975; 94: 15-22.

18 Tobey $\mathrm{RA}$, Ley $\mathrm{KD}$. Isoleucine-mediated regulation of genome replication in various mammalian cells lines. Cancer Res 1971; 31: 46-51.

19 Zimber A, Visek WJ. Effect of urease injections on DNA synthesis in mice. Am $\mathcal{F}$ Physiol 1972; 223: 1004-8. 